\title{
Breast cancer - review of complementary and alternative medicine (CAM) and traditional chinese medicine (TCM) approach
}

\begin{abstract}
In this article, we reviewed currently breast cancer risk factors, diagnosis and therapeutic opportunity in conventional medicine and complementary/alternative medicine (CAM) as well as Chinese Traditional Medicine (CTM), summarized different views on breast cancer between two medicines and application of utilizing CAM/CTM in treatment of breast cancer. In old CTM book described breast cancer as "Yan" and considered it is significantly related to emotional change special depression and lifetime hardship. In early stages, acupuncture and herb medicine can be considered as alternative medicine for treatment. Exercise to maintain good emotion can be considered as an effective way to prevent from breast cancer. However, better-designed trials of CAM/CTM methods on breast cancer prevention, progression, treatment and outcomes are needed in order to standardize their use as we move towards the personal medicine approach. Regular screen breast cancer in conventional medicine and early stage treatment is important.
\end{abstract}

Keywords: breast cancer, CAM, TCM, Qi-Gong, yoga, supplement, acupuncture, moxibustion
Volume I Issue 3 - 2015

\author{
Alvaro Menendez,' Luguang Luo',2 \\ 'Roger Williams Medical Center/Boston University School of \\ Medicine, Providence, USA \\ ${ }^{2}$ The Center for Natural Healing, Rhode Island, USA \\ Correspondence: Dr. Luguang Luo, Prior Building Floor 2, \\ Center for Stem Cell Research, Department of Medicine/ \\ Research, Roger Williams Hospital, Chartercare LLC, 825 \\ Chalkstone Avenue, Providence, Rhoda Island 02908, USA, Tel \\ 40I4565344,Email lluo@chartercare.org
}

Received: May 05, 2015 | Published: July 15, 2015

\section{Introduction}

Breast cancer constitutes the second leading cause of cancer deaths among women. It is estimated that in 2015 a total of 40,730 related deaths will occur. ${ }^{1,2}$ Established risk factors for this disease include age in a cumulative fashion, breast density, reproductive and/or menstrual history, use of oral contraceptives and/or hormone replacement therapy, radiation exposure, alcohol intake, physical activity and anthropometry. ${ }^{3,4} \mathrm{~A}$ genetic contribution in an autosomal dominant inheritance with high penetrance as also been established. In the general population however they are estimated to account for no more than $10 \%$ of breast cancer cases. ${ }^{5}$ It is therefore likely that other genetic factors contribute to the etiology of this cancer., The syndromes most strongly associated with breast cancer are the BRCA1 and BRCA2 mutation syndromes, although it can also be associated with Li-Fraumeni syndrome, Cowden syndrome, ataxia telangiectasia and Peutz-Jeghers syndrome. ${ }^{8}$ It's been estimated that women with a mutated BRCA1 or BRCA2 gene have up to an $85 \%$ risk of developing breast cancer by age 70, although little is known about how cancer risks differ between BRCA1 or BRCA2 mutation type.

\section{Preventing, diagnosing and treating breast cancer with conventional medicine}

Increased public awareness and screening rates have led to earlier diagnosis and improved survival rates. ${ }^{9}$ Screening and prevention guidelines for breast cancer rely on a combination of factors involving age and evidence about the risk of the condition, benefits, harms and cost, with mammography remaining the mainstay of screening and ultrasonography reserved to clarify features of a potential lesion. Monthly breast self-examination is recommended to begin at age 18 , although it remains unclear if this provides a mortality benefit. ${ }^{10}$ Most American groups recommend screening with mammography with or without clinical breast examination starting at age 50. Controversy persists about routine screening for women in their $40 \mathrm{~s},{ }^{11}$ with an increasing number of institutions recommending it despite of high risk for false-positive results and unnecessary work-up. ${ }^{12,13}$ Additionally patients with breast cancer gene mutations are counseled to initiate screening earlier and at more frequent intervals than the general population. ${ }^{14,15}$ Clinical breast examination a minimum of 2 times a year beginning at age 25 , accompanied by annual mammography and breast magnetic resonance imaging should complete their screening. ${ }^{16,17}$

More recently, chemoprevention with Tamoxifen, Raloxifene or Exemestane has proven capable of reducing the incidence of this disease by approximately $50 \%$ in women with a moderate risk for breast cancer, although studies are inconclusive when analyzing disease-specific and all-cause mortality and no specific data has been obtained in patients with BRCA mutations. Risk-reducing surgery consisting of prophylactic bilateral mastectomy can reduce the risk of developing breast cancer by a minimum of $90 \%{ }^{18}$

Following diagnosis, this pathology is treated with a multidisciplinary approach involving surgical oncology, radiation and medical oncology. ${ }^{19}$ The vast majority of patients with newly diagnosed breast cancer in the United States and developed countries have no evidence of metastatic disease with only $5 \%$ of them having de novo widely metastatic disease at the time of presentation. Surgery and radiation therapy, along with adjuvant hormone or chemotherapy when indicated, are now considered primary treatment for early breast cancer. However, despite the advances made in conventional treatment modalities, these still remain costly and can be the cause for long-term morbidity and mortality. Special attention has therefore been paid to other types of treatment, especially those that increase patient's comfort levels, quality of life and even maybe mortality. ${ }^{20}$ This review offers an insight into the history and potential benefits of TCM and CAM for the prevention and treatment of breast cancer. 


\section{Complementary and alternative medicine}

CAM is referred to as a "group of philosophies, diagnostic and therapeutic interventions that are not part of conventional or regulated methods of a particular society". ${ }^{21}$ The National Center for Complementary and Alternative Medicine goes beyond and broadens the classification into those based on biology, "mind and body", energy and practices of bodily manipulation. ${ }^{22}$ Among all the practices in CAM, the use of dietary supplements, including herbal and non-herbal supplements, is by far the most common. Some of the other frequently used modalities in CAM include but are not limited to spirituality, meditation, acupuncture, and yoga although a recent study proved that more frequently than not, these are all used in combination with one another. ${ }^{23}$

Meditation can be classified as either transcendental or mental. The first one is based on the repetition of a specific mantra with the purpose of silencing and transcending the mental dialogue of the patient. The latter pretends to develop an objective purpose or role toward their emotions, feelings, perceptions, etc. As a general concept, meditation itself has been proven to decrease anxiety, depression, anger and confusion as well as stress in patients undergoing conventional oncological treatment with curative or palliative purposes. ${ }^{24}$

On the other hand, yoga dates back to pre-Vedic Indian traditions although it was only introduced to the west following the success of Swami Vivekananda in the late 19th and early 20th century. ${ }^{25}$. Initially considered a system of physical exercise, its meditative and spiritual core have made it increasingly more popular for the treatment of breast cancer symptoms and complications, and sometimes even for the side effects caused by its treatment. Many studies have tried to determine the effectiveness of yoga as a complementary intervention for cancer with mixed and inconclusive results and some even suggesting no effectiveness with the exception of its capacity to aid a patient's psychological healing process, stress, anxiety and insomnia, ${ }^{26}$ a beneficial result if it's used for alternative or palliative purposes, although the population studied may not be equivalent to a palliative care population.

Dietary supplements popularity has progressively increased over the last couple of decades. ${ }^{20}$ These include, but are not limited to, vitamin $\mathrm{A}$, vitamin $\mathrm{C}$, vitamin $\mathrm{E}$, green tea, soy, grape seed extract, melatonin and selenium. Hildenbrand GL et al. ${ }^{27}$ worked under the theory that the Gerson regimen, a modality based on low sodium intake, high potassium and a lactovegetarian diet emphasizing fresh vegetables, fruit juices and vitamin supplements, was beneficial in increasing survival rates in patients diagnosed with certain cancers. ${ }^{27}$ Other nutritional modalities studied macrobiotic diets (a high-complex carbohydrate, low fat vegetarian diet) ${ }^{28}$ and the Gonzalez NJ \& Isaacs LLO. ${ }^{29}$ regimen, which consists of dietary restriction and increased intake of digestive aids such as pepsin and pancreatic enzymes in combination with a detoxification regimen that includes frequent coffee enemas. ${ }^{29}$ The use of herbal supplements such as TCM, green tea, mistletoe, Essaiac, PC-SPES (Prostate Cancer and "SPES" Latin for Hope), Sho-saiko-to, Saint John's worts and Astralagus has been studied. Significant methodological limitations and contradictory results in all of the trials studying their benefits have prevented integration to conventional oncologic treatment. ${ }^{30}$ For instance, TCM did not prove beneficial at all for the hematologic and non-hematologic toxicity of medical treatment with the exception of the nausea component of patients with breast cancer undergoing standardized treatments. On the other hand, non- herbal dietary supplements extend through melatonin, shark and bovine cartilage, thymus extracts, hydrazine and Coenzyme Q10, the latter working under the assumption that it might improve tolerance for chemotherapy. ${ }^{31}$ As a general principal, most of the non-herbal supplements have shown some hematologic and non-hematologic benefit but results have not been reproducible, so their use should not be systematic. Moreover, concerns about unwanted or polarizing side effects have surfaced. For instance, some of these supplements prevent cellular oxidative changes in the cells completely antagonizing the effect of radiotherapy and therefore potentially jeopardizing success of conventional oncological treatment. Contradictory statements are now coming up since some believe that these modalities can actually act as synergistic agents to the scientific standardized protocols.

\section{Traditional Chinese medicine}

TCM has been around for more than five thousand years and it has been used alongside standard cancer treatments to reduce treatment toxicity and to improve treatment outcomes, performance status and quality of life, its mechanism of action and impact on mortality however remain unclear. The doctrines of Chinese medicine are rooted in books such as the "Yellow Emperor's Inner Canon" and the "Treatise on cold Damage", as well as in cosmological notions like "Yin-Yang" and the "Five phases". Nowadays these precepts have been integrated into some anatomical and pathological notions along with modern scientific medicine. Conversely most of them, including the model of the body or concept of disease, are still not supported by modern evidence-based medicine. This is further evidenced by contrasting ignorance and incompatibility between both worlds, where most of TCM providers barely known what experimental western medicine is and where conventional medical providers underanalyze TCM's impact in disease outcomes. For instance a meridian theory was developed more than two thousand years ago that describes energy flow through tracks that fit exactly the hypothesized Meridian (Qi) distribution. Appropriate methodology is needed to be created for conventional medicine, further evidencing the discordance between both worlds.

The significant difference between TCM and conventional medicine is that the first one's view of the body mainly concerns the identification of functional entities (i.e. digestion, breathing, aging, etc.) with little emphasis on anatomical structures. Health is perceived as harmonious interaction of these entities and the outside world with disease resulting as consequence of disharmony. As such, TCM considers that supporting body function and survival is through fluent energy and interchangeable energy with mass. In the case of breast pathology for instance (calcifications, cysts or tumor), it can be interpreted as stagnant energy that transformed into masses. When energy reestablishes free flow in the body, these breast conditions could potentially resolve themselves.

Breast cancer cases have been recorded in various TCM books. The Famous Dr. Dan Qi Zhu (1281 1358 AD) recorded a case as following: "one's wife does not enjoy a good relationship with her husband, nor does she have good relationships with family members and others, then chronic anger, depression, worry and nervousness accumulates in her daily. Without treatment, this condition causes spleen Qi or 'digestive system' disorder and liver Qi stagnation. Eventually, this causes a small lump in the breast that is without pain and itchiness. Many years later, this lump turns into a different shape (like a craggy rock with many holes), which is called 'Yan' or cancer. If the lump acquires this shape, it is very difficult to fix". Another 
record from Dr. Chen in his Wai Ke Zhang Zong (1400 AD) states, "Negative emotions accumulate day by day and cause spleen and stomach Qi deficiency and liver Qi stagnation. These conditions will cause the body to create a lump. When Qi stagnation accumulates in the meridians over time, a small seed in the breast can progress to a cancerous mass. Then the five major organs will spiral out of balance. This problem is called cancer." 32

\section{Emotion is a significant contributor to breast cancer}

According to TCM principles, emotions can influence one's health. TCM books elaborated on them and categorized them as "seven affections" and "six desires", or "endogenous factors", which include lust, vanity, dignity, pleasant sounds, good life/death and sensual pleasures. Both "affections" and "desires" are established contributors of disease. The seven emotions include joy, anger, grief, worry, fear, sentiments and affection, and they constitute physiological reflections of the human mental state inducible by various environmental stimulations. Under normal conditions these usually do not cause diseases. However, if they are too stressful and constant, or if the patient is too sensitive to them, they may induce acute and long-standing pathologies. They are also capable of disturbing the functional activities of Qi. For example, according to an ancient saying, "Anger makes the Qi rush upward, over-joy makes the Qi circulate slowly, grief consumes it, fear causes it to flow downward, fright makes Qi flow disorderly, and over-thinking leads to Qi stagnation". Furthermore over-activation of these can selectively damage certain "zàng" or "fŭ" organs. For instance, anger injures the liver, over-joy injures the heart, grief and melancholy injure the lung, over-thinking injures the spleen, and fright and fear injure the kidney. The zàng-fŭ (simplified Chinese: 脏腑, traditional Chinese: 臟腑) organs are functional entities stimulated by TCM. They constitute the centre piece of TCM's general concept of how the human body works. The term zàng (脏) refers to the organs considered to be yin in nature such as the heart, liver, spleen, lung and kidney, while fŭ (腑) refers to the yang organs which include the small and large intestine, the gallbladder, the urinary bladder and the stomach. Each zàng is paired with a fú and each pair is assigned to one of the Wǔ Xíng. The zàngfurs are also connected to the twelve standard meridians with each yang meridian attached to a fŭ organ and each yin meridian attached to a zàng. Together they form five systems: Heart, Liver, Spleen, Lung, Kidney. The TCM book Lingshu says, "The heart is the monarch of the five zàng and six fŭ organs, therefore, grief and melancholy also disturb the heart and a disturbance of the heart leads to the affection of the five zàng and six fǔ organs”. Therefore, even though emotional pathogenic factors can injure either one of the five zàng, they are all capable in the end of injuring the heart.

Qigong (simplified Chinese: 气功, Traditional Chinese: 氣功) means "Life Energy Cultivation" and is a type of spiritual practice intended to align the body, breath and mind for health, meditation and martial arts training. With roots in TCM, philosophy and martial arts, Qigong is viewed as a mean to cultivate and balance Qi. By doing so, higher realms of awareness are reached while awakening one's "true nature" and developing the human potential. Qigong practices typically involve meditation, deep rhythmic breathing and calm meditative state of mind. The Chinese Qigong may affect the endocrinological system by changing glucocorticoid receptor on the human lymphocyte cells and to other hormones such as epinephrine and Norepinephrine to potentially aid people during cancer recovery. ${ }^{33}$
Based on these observations, we conclude that maintaining good emotion is critical to prevent breast cancer. When treating patients, TCM always includes liver Qi calming techniques in order to prevent stagnancy. Additionally, TCM ensures good emotion will allow patients to prevent breast cancer. This is achieved through acupuncture and relaxation.

\section{Acupuncture and moxibustion}

Acupuncture is one of most interesting treatment tools of TCM. With a small needle inserted in certain areas of the body, pain is relieved and the body's healing is facilitated. Acupuncture, and its derivative, eletroacupunture, originated from Chinese, Japanese, Korean and French theories and techniques. Although their role in mortality benefit for breast cancer remains to be determined, several authors have argued that there is already enough evidence for acupuncture and / or acupressure to be included as an important modality within palliative care. ${ }^{34}$

A study performed in 1997 during the Acupuncture Conference concluded that such practices contributed to better management of nausea and vomiting, and odontological pain induced by chemotherapy. The study also stated that unwanted side effects related to these techniques were only limited to minimal bleeding and mild pain on the insertion site. ${ }^{35}$ Although its mechanism of action is still unknown, its popularity has progressively increased and is now an important part in the treatment of multiple diseases. Based on current evidence and scientific knowledge, the potential mechanisms of acupuncture have been proposed in the following models:

Biological compute model: It has been proposed that the human body acts as a biological computer. Organs are the headquarters and the body surface is the keyboard. Both of them are wired through proposed meridians (Ying and Yang just like electrical circulation Negative pole (ying) and positive pole (Yang)). When a body is alive, a keyboard can be used to send instructions through twelve meridians and other Jing/Lou (additional channels) to the organs initiating an action. This can be easily witnessed, for example, when a patient's abdominal pain is treated and stomach or intestines start moving after the "keyboard" has been tapped through the acupuncture needles.

Energy following model: Using a physic device to detect energy flow or positive messages originated from insertion of an acupuncture needle on the body surface, a distribution of it can be mapped just like a meridian. A patient with severe shoulder pain was initially treated in points of the "small intestine" meridian which distribute to the shoulder area with some improvement. Complaint only completely disappeared when "bladder" meridian points, which connect to pain area, were targeted. Detecting energy flow points is a significant contribution for acupuncture theory and will give a direction for further standard therapeutic modalities development.

ATP/ADP transition model: Molecular biological studies show that acupuncture manipulation of certain points induces significant ATP production increase. Once released, ATP acts as a transmitter that binds to purinergic receptors, including $\mathrm{P} 2 \mathrm{X}$ and $\mathrm{P} 2 \mathrm{Y}$ receptors. ATP cannot be transported back into the cell but is rapidly degraded to adenosine by several ectonucleotidases before re-uptake. Thus, adenosine acts as an analgesic agent that suppresses pain through Gicoupled A1-adenosine receptors. ${ }^{36}$ 
Connective tissue stretch theory: Connective tissue (CT) supports, connects, or separates different types of tissues and organs of the body. It is one of the four general classes of biological tissues. All CT has three main components: cells, fibers, and extracellular matrices, all immersed in the body fluids. Connective tissue can be broadly subdivided into proper connective tissue, special connective tissue, and a series of other less classifiable types of connective tissues. Proper connective tissue consists of loose connective tissue and dense connective tissue, and special connective tissue consists of reticular connective tissue, adipose tissue, cartilage, bone and blood. The mysterious acupuncture meridians may also be related to connective tissue, as they appear to be preferentially located along connectivetissue planes between muscles or between muscle and bone. Authors have found that more than 80 percent of acupuncture points in the arm are located along connective-tissue planes. This makes sense, since loose connective tissue houses blood vessels and nerves, suggesting that mechanical stimulation of connective tissue generated by needle manipulation could transmit a mechanical signal to sensory nerves, as well as intrinsic sensory afferents directly innervating connective tissue. Acupuncture-needle manipulation results in sustained stretching and therefore constitutes a useful tool that can be used to study the acupuncture biomechanical function. A twisted acupuncture needle creates a localized stretch by gripping the underlying connective tissue. This effect can be observed as a "tenting" of the skin as the needle is pulled out. ${ }^{37}$

Immunological reaction model: acupuncture needles can be perceived as foreign material causing the body to immediately initiate an immune system response. Even after the removal of the needle withdrawal, this immune reaction continues to work locally until disorder is eliminated or controlled.

Physics quark particular-string theory: It unveils an explanation of supersymmetry of particles by string correlation. Any force acts on a particular quark to induce a similar movement in peer quarks in unlimited distance. Since string theory incorporates all of the fundamental interactions, including gravity, many physicists hope that it fully describes our universe, making it a theory of everything. Current research in string theory is focused in finding a solution that is quantitatively identical with the standard model, along with a small cosmological constant, containing dark matter and a plausible mechanism for cosmic inflation. Particular Quark is found to fit string theory which has been described as peer particular linked with string in unlimited distances. In our clinical and others found stimulating a distant point for pain of paired area in body to have significantly impact for distance paired area's pain relief which just likes to move a particular quark to induce a similar movement in peer quarks in unlimited distance. This model still needs a significantly works to approve it.

Moxibustion is another special tool of TCM and dates back to the 16th century. Artemisia, or mugworth, (ài (艾)or àicăo (艾草) in Chinese) is an herb used to produce "Moxa" (jiǔ (尒) or jiǔshù (尒術). This is used to warm meridian points in the body with the intention of stimulating circulation and inducing a smoother Qi. Some believe it can treat conditions associated with the "cold" or "yang deficiencies" as described in TCM. Bian Que, one of the most famous semi-legendary doctors of TCM and the first specialist in moxibustion, discussed the benefits of moxa over acupuncture in Bian Que Neijing. He asserted that moxa could add new energy to the body and could treat not only deficient conditions but also "excesses". As such, if cancer patient feels weak or in "cold", moxibustion treatment may provide a beneficial effect. However, other conditions may have not such benefits so that further identification of well designed clinical trials would be useful for moxibustion in breast cancer treatment.

\section{Conclusion}

Clinical breast exam and frequent mammographies with MRI or other imaging techniques when indicated remains unsurpassed for early detection of breast cancer. TCM and CAM, including acupuncture and moxibustion, are increasingly becoming an alternative to treat concomitant pathologies and/or breast cancer treatment-related sideeffects, and may even offer mortality benefits in some of these patients. There is evidence that some of these therapies may well be of clinical benefit in a palliative care population as well. More importantly, their role in preventing this disease and its progression is now being recognized. Better designed trials of these methods on breast cancer prevention, progression, treatment and outcomes are needed in order to standardize their use as we move towards the integrative medicine approach.

\section{Acknowledgments}

None.

\section{Conflict of interest}

The authors declare that there is no conflict of interest.

\section{Funding}

None.

\section{References}

1. American Cancer Society. Cancer Facts and Figures 2015. Atlanta, USA: American Cancer Society; 2015. p. 1-52.

2. Siegele RL, Miller KD, Jemal A. Cancer statistics, CA Cancer J Clin. 2015;65(1):5-29.

3. NIH. Genetics of Breast and Gynecologic Cancers-for health professionals (PDQ $\left.{ }^{\circledR}\right) .2015$.

4. Nelson HD, Zakher B, Cantor A, et al. Risk factors for breast cancer for women aged 40 to 49 years: a systematic review and meta-analysis. Ann Intern Med. 2012;156(9):635-648.

5. Foulkes WD. Inherited susceptibility to common cancers. $N$ Engl J Med. 2008;359(20): 2143-2153.

6. Colditz GA, Willett WC, Hunter DJ, et al. Family history, age, and risk of breast cancer. Prospective data from the Nurses' Health Study. JAMA. $1993 ; 270(3): 338-343$.

7. Johnson N, Lancaster T, Fuller A, et al. The prevalence of a family history of cancer in general practice. Fam Pract. 1995;12(3):287-289.

8. Lindor NM, McMaster ML, Lindor CJ, et al. Concise handbook of familial cancer susceptibility syndromes - second edition. J Natl Cancer Inst Monogr. 2008;38:1-93.

9. Jemal A, Siegel R, Ward E, et al. Cancer statistics 2006. CA Cancer J Clin. 2006;56(2):106-130.

10. National Comprehensive Cancer Network (NCCN). NCCN Clinical practice guidelines in oncology.

11. van Ravesteyn NT, Miglioretti DL, Stout NK, et al. Tipping the balance of benefits and harms to favor screening mammography starting at age 40 years: a comparative modeling study risk. Amm Intern Med. 2012;156:609-617. 
12. Lee CH, Dershaw DD, Kopans D, et al. Breast cancer screening with imaging: recommendations from the Society of Breast Imaging and the ACR on the use of mammography. J Am Coll Radiol. 2010;7(1):18-27.

13. Smith RA, Cokkinides V, Brawley OW. Cancer screening in the United States, 2009:a review of current American Cancer Society guidelines and issues in cancer screening. CA Cancer J Clin. 2009;59(1):27-41.

14. Daly MB, Axilbund JE, Buys S, et al. Genetic/familial high risk assessment: breast and ovarian. J Natl Compr Canc Netw. 2010;8(5):562594.

15. Kerlikowske K, Zhu W, Hubbard RA, et al. Outcomes of screening mammography by frequency, breast density, and postmenopausal hormone therapy. JAMA Intern Med. 2013;173(9):807-816.

16. Saslow D, Boetes C, Burke W, et al. American Cancer Society guidelines for breast screening with MRI as an adjunct to mammography. $C A$ Cancer J Clin. 2007;57(2):75-89.

17. Le-Ptross HT, Wjitman GJ, Atchley DP, et al. Effectiveness of alternating mammography and magnetic resonance imaging for screening women with deleterious BRCA mutations at high risk of breast cancer. Cancer. 2011;117(17):3900-3907.

18. Rebbeck TR, Friebel T, Lynch HT, et al. Bilateral prophylactic mastectomy reduces breast cancer risk in BRCA1 and BRCA2 mutation carriers:the PROSE Study Group. J Clin Oncol. 2004;22(6):1055-1062.

19. Kesson EM, Allardice GM, George WD, et al. Effects of multidisciplinary team working on breast cancer survival:retrospective, comparative, interventional cohort study of 13722 women. BMJ. 2012;344:e2718.

20. Eisenberg DM, Davis RB, Ettner SL, et al. Trends in alternative medicine use in the United States, 1990-1997. Results of a follow-up national survey. JAMA. 1998;280(18):1569-1575.

21. Panel on Definition and Description, Office of Alternative Medicine, National Institutes of Health, CAM Research Methodology Conference, April 1995. Altern Ther Health Med. 1995;3(2):49-57.

22. http://nccam.nih.gov/plans/2005/inde8.htm

23. Menendez A MD, Said Calvino A MD, Espat J MD . "Patient Reported Experience Combining Complementary and Alternative Medicine (CAM) with Conventional Oncology Treatment (COT)". J Clin Oncol. 2015;33(suppl; abstr 9625).

24. Speca M, Carlson LE, Goodey E, et al. A randomized, wait-list controlled clinical trial: the effect of a mindfulness meditation-based stress reduction program on mood and symptoms of stress in cancer outpatients. Psychosom Med. 2000;65(2):613-622.
25. Gordon WD. Yoga, Brief History of an Idea (Chapter 1 of "Yoga in practice") (PDF). Princeton, USA: Princeton University Press; 2011. p. $1-23$.

26. Ott MJ. Complementary and alternative therapies in cancer sympton management. Cancer Pract. 2002;10(3):162-166.

27. Hildenbrand GL, Hildenbrand LC, Bradford K, et al. Fie-year survival rates of melanoma patients treated by diet therapy after the manner of Gerson:a retrospective review. Altern Ther Health Med. 1995;1(4):2937 .

28. Carter JP, Saxe GP, Newbold V, et al. Hypothesis:dietary management may improve survival from nutritionally linked cancers based on analysis of representative cases. J Am Coll Nutr. 1993;12(3):209-226.

29. Gonzalez NJ, Isaacs LLO. Evaluation of pancreatic proteolytic enzyme treatment for adenocarcinoma of the pancreas, with nutrition and detoxification support. Nutr Cancer. 1999;33(2):117-124.

30. Mok TS, Yeo W, Johnson PJ, et al. A double-blind placebo-controlled randomized study of Chinese herbal medicine as complementary therapy for reduction of chemotherapy-induced toxicity. Ann Oncol. 2007;18(4):768-774.

31. Roffe L, Schmidt K, Ernst E. Efficacy of coenzyme Q10 for improved tolerability of cancer treatments:a systematic review. $J$ Clin Oncol. 2004;22(21):4418-4424.

32. Understanding breast cancer from the traditional chinese medicine perspective. Traditional Chinese medicine.

33. Luo L-G, Tao Xingming, DuFengming. The effect of Chinese Qi Gong on glucocorticoid receptor of human lymphocyte and some hormones in the patient with cancer. Chinese Journal of Qi Gong. 1989;10(2):89-91.

34. MacPherson H, Thomas K, Walters S, et al. The York acupunture safety study:a prospective survey of 34000 treatments by aditional acupunturists. BMJ. 2011;323(7311):486-487.

35. Pan CX, Morrison RS, Ness J, et al. Complementary and alternative medicine in the management of pain, dyspnea, and nausea and vomiting near the end of life. A systematic review. J Pain Symptom Manage. 2011;20(5):374-387.

36. Burnstock G. Acupuncture:A novel hypothesis for the involvement of purinergic signaling. Med Hypotheses. 2009;73(4):470-472.

37. Langevin HM. The Science of Stretch - The study of connective tissue is shedding light on pain and providing new explanations for alternative medicine. The Scientist. 2013. 